
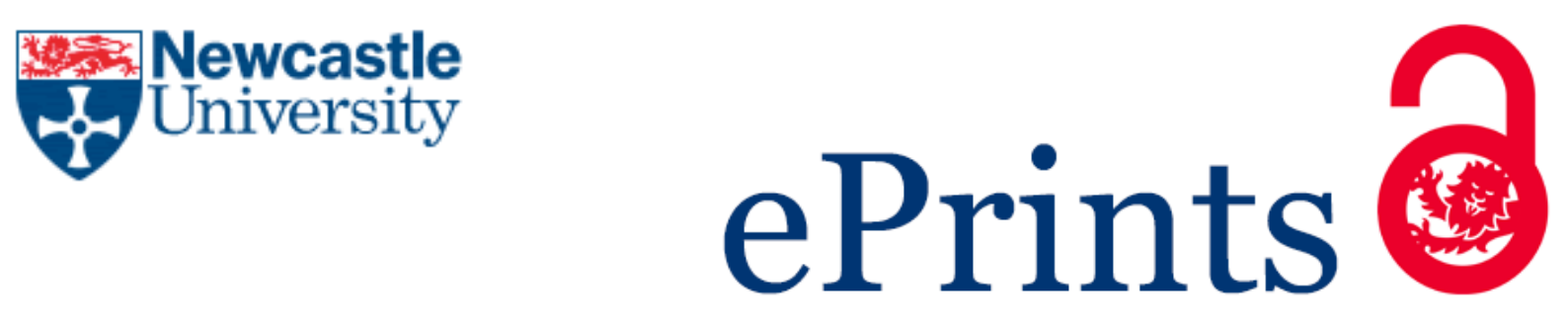

Firbank MJ, Watson R, Mak E, Aribisala B, Barber R, Colloby SJ, He J, Blamire AM, O'Brien JT.

Longitudinal diffusion tensor imaging in dementia with Lewy bodies and

Alzheimer's disease.

Parkinsonism and Related Disorders, 2016

DOI: http://dx.doi.org/10.1016/j.parkreldis.2016.01.003

\title{
Copyright:
}

(C) 2016. This manuscript version is made available under the CC-BY-NC-ND 4.0 license

DOI link to article:

http://dx.doi.org/10.1016/i.parkreldis.2016.01.003

Date deposited:

$06 / 01 / 2016$

Embargo release date:

06 January 2017

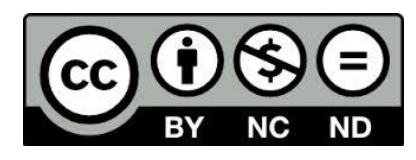

This work is licensed under a

Creative Commons Attribution-NonCommercial-NoDerivatives 4.0 International licence 


\section{Longitudinal diffusion tensor imaging in dementia with Lewy bodies and Alzheimer's disease}

Michael J. Firbank,PhD ${ }^{1}$ Rosie Watson, PhD $^{1,2}$ Elijah Mak,BA ${ }^{3}$ Benjamin Aribisala,PhD ${ }^{5,6}$ Robert Barber, $\mathrm{MD}^{1}$ Sean J. Colloby, $\mathrm{PhD}^{1}$ Jiabao He,PhD ${ }^{4}$ Andrew M. Blamire,PhD John T. O’Brien, $\mathrm{DM}^{3}$

1 Institute for Neuroscience and Newcastle University Institute for Ageing, Campus for Ageing and Vitality, Newcastle University, Newcastle upon Tyne, UK

2 The Florey Institute of Neuroscience and Mental Health, The University of Melbourne, Parkville, Australia and The Department of Aged Care, The Royal Melbourne Hospital, Parkville, Australia.

3 Department of Psychiatry, University of Cambridge School of Clinical Medicine, Box 189, Level E4, Cambridge Biomedical Campus, Cambridge, UK

$4 \quad$ Aberdeen Biomedical Imaging Centre, University of Aberdeen, UK

5 Newcastle Magnetic Resonance Centre, Campus for Ageing and Vitality, Newcastle University, Newcastle upon Tyne, UK

6 Computer Science Department, Faculty of Science, Lagos State University, Lagos, Nigeria

Corresponding author: Michael Firbank michael.firbank@ncl.ac.uk

Institute of Neuroscience

Newcastle University

Campus for Ageing and Vitality

Newcastle upon Tyne,

NE4 5PL

Tel: +44 (0)1912081319

Study funding: The study was funded by the Sir Jules Thorn Charitable Trust (grant ref: 05/JTA) and was supported by a UK NIHR Biomedical Research Centre for Ageing and Age-Related Disease Award to the Newcastle upon Tyne Hospitals NHS Foundation Trust (grant ref: Newcastle BRC). 
Financial Disclosure: John O'Brien has been a consultant for GE Healthcare, Servier, and Bayer Healthcare and has received honoraria for talks from Pfizer, GE Healthcare, Eisai, Shire, Lundbeck, Lilly, and Novartis. All other authors have no conflicts of interest to declare.

Running title: Longitudinal DTI changes in AD and DLB

Key words: Dementia with Lewy bodies ; Alzheimer's Disease; DWI ; MRI 
ABSTRACT

\section{Objective}

Changes in the white matter of dementia with Lewy bodies (DLB) and Alzheimer's disease (AD) have been reported using diffusion weighted MRI, though few longitudinal studies have been done.

\section{Methods}

We performed diffusion weighted MRI twice, a year apart on 23 AD, 14 DLB, and 32 healthy control subjects. Mean diffusivity (MD) and fractional anistropy (FA) were calculated.

\section{Results}

In $A D$, there were widespread regions where the longitudinal MD increase was greater than in controls, and small areas in the parietal and temporal lobes where it was greater in AD than DLB. In $A D$, decrease in brain volume correlated with increased MD. There were no significant differences in progression between DLB and controls.

\section{Conclusions}

In $A D$ the white matter continues to degenerate during the disease process, whereas in DLB, changes in the white matter structure are a relatively early feature. Different mechanisms are likely to underpin changes in diffusivity. 


\section{OBJECTIVE}

Dementia with Lewy bodies (DLB) is a common form of neurodegenerative dementia in older people, second to Alzheimer's disease (AD). Studies of DLB have found relatively less overall brain atrophy than $A D$, particularly in the temporal lobes.[1] A number of studies have investigated the structural integrity of the white matter in DLB using MR diffusion tensor imaging (DTI) and have found mixed results, but generally, DLB subjects have DTI changes in parieto-occipital regions, whilst $A D$ changes are more widespread, and have been reported to particularly involve the medial temporal lobe and limbic pathways.[1, 2]

In a previous study, we found widespread reductions of fractional anisotropy (FA) in AD, with DLB changes more restricted to parieto-occipital regions, along with generally increased mean diffusivity (MD) in both groups.[3] In this study, we report the longitudinal change in MD and FA measured over 1 year in these subjects.

\section{METHODS}

\section{Subjects}

Seventy-one individuals aged over 60 (36 subjects with probable AD[4] and 35 with probable DLB[5]) were recruited from a community-dwelling population of patients referred to local old age psychiatry, geriatric medicine, or neurology services. Thirty-five control subjects were recruited from relatives and friends of subjects with dementia or volunteered via local advertisements. All subjects underwent clinical and neuropsychological evaluations with diagnostic procedures as previously described.[3] MR scanning was done on all subjects at baseline and was repeated after 1 year on those subjects who consented for repeat scanning. The research was approved by the local ethics 
committee. All subjects or, where appropriate, their nearest relative, provided written informed consent.

Of the $36 \mathrm{AD}$ subjects, 23 were included after 11 were unable to participate in the follow-up assessment, one AD was excluded due to excessive motion on the repeat DT images, and DTI data were not available on another. Of the 35 DLB subjects, 14 were included after 12 declined to participate due to ill health, and 9 subjects had died. Of the $35 \mathrm{HC}$ subjects, 32 were included in the present analyses after 2 declined to participate, and DTI data were not available on one control.

Assessment of global cognitive measures in all subjects (those with AD or DLB and controls) involved use of the Cambridge Cognitive Examination (CAMCOG), which incorporates the Mini-Mental State Examination (MMSE).[6] Verbal episodic memory and visual episodic memory were assessed with the Hopkins Verbal Learning Test (HVLT) and Brief Visual Memory Task-Revised (BVMT), respectively, and a composite z-score of episodic memory (verbal and visual) relative to control group performance was derived to incorporate all aspects of the memory task, as described previously.[3]

Verbal fluency was assessed with the Delis Kaplan letter fluency task (FAS). Motor parkinsonism was assessed with the Unified Parkinson's Disease Rating Scale Part III (UPDRS-III).[7]

\section{Imaging}

Subjects underwent MRI scanning on a 3T MRI system (Intera Achieva scanner, Philips Medical Systems, Eindhoven, Netherlands) with an 8-channel receiver head coil within 2 months of the baseline cognitive study assessment, and at 1 year after the initial scan.

DTI images were acquired using a Pulsed Gradient Spin Echo (PGSE) sequence and multi-slice single shot EPI readout, with 24 slices. TE=71 ms and TR=2524 ms with $2 \mathrm{~mm}$ in-plane resolution, $6 \mathrm{~mm}$ 
slice thickness and matrix size of $128 \times 128$. Diffusion weighting was in 16 directions with a b value of $1000 \mathrm{~s} . \mathrm{mm}^{-2}$ and a single measurement at $b=0 \mathrm{~s} . \mathrm{mm}^{-2}$.

A T1 weighted volumetric sequence (3D MPRAGE, sagittal acquisition, $1 \mathrm{~mm}$ isotropic resolution and matrix size of $240 \times 240 \times 180 ; \mathrm{TR}=9.6 \mathrm{~ms} ; \mathrm{TE}=4.6 \mathrm{~ms}$; flip angle= $8^{\circ}$; SENSE factor=2) was also acquired. Additionally, we acquired quantitative images using a fast quantitative T1 measurement based on a custom inversion recovery prepared EPI sequence (axial slices, $2 \mathrm{~mm}$ isotropic resolution, matrix $128 \times 128 \times 72, T R=15 \mathrm{~s}, \mathrm{TE}=24 \mathrm{~ms}$, inversion time $\mathrm{TIR}=0.25-2.5 \mathrm{~s}$ in 12 uniform steps); and a fast quantitative multi-echo T2 measurement based on a Gradient and Spin Echo Imaging sequence ( $2 \mathrm{~mm}$ isotropic resolution, matrix $128 \times 128 \times 72, \mathrm{TR}=4.7 \mathrm{~s}, 8$ spin echoes at $20 \mathrm{~ms}$ spacing, EPI factor 5).

\section{Image processing}

Data were processed with the FSL toolbox (http://fsl.fmrib.ox.ac.uk/fsl/fslwiki/). The DTI images were processed to correct for subject motion and eddy current distortion, and fractional anisotropy (FA) and mean diffusivity (MD) calculated. The baseline and 1 year FA images were spatially registered together, by calculating the transform from base to 1 year, and vice versa, and resampling both images to the space halfway between them. The average of the baseline and 1 year FA maps was then calculated, along with the difference of the two MD and FA maps. We then used the TBSS package in FSL to align the average FA maps for each subject to the standard template, and to create a mean FA skeleton representing the centres of all tracts common to the cohort. Each subject's difference FA and MD data were then projected onto the mean FA skeleton. Voxelwise statistics on the skeleton were then performed on the difference images to look for longitudinal increases or decreases in FA and MD between groups. This was done as an ANOVA, without covariates, and also with the addition of age, sex, and WMH score to the model. We used the TFCE (threshold-free 
cluster enhancement)[8] to find regions of significant difference between groups correcting for multiple comparisons. We also calculated the mean (and SD) of change in FA and MD within the entire skeleton for each group. Reported clusters are all $p<0.05$ corrected for multiple comparisons. In addition, we used the FSL SIENA package to determine longitudinal brain atrophy from the 3D T1 weighted images, as described in our previous publication.[9]

In order to visualise white matter hyperintensities, an image with contrast resembling a FLAIR (fluid attenuated inversion recovery) sequence was calculated from the quantitative T1 and T2 maps [10] and the standard image contrast for inversion recovery. [11] The resulting images (see supplementary figure e1 for examples) were reviewed by two experienced raters (RB, SJC) who rated the white matter hyperintensities according to the Fazekas scale. [12] We took the deep WMH score as representative of vascular disease and included it in the analysis of the DTI data.

\section{STATISTICS}

We used SPSS (version 22, IBM, New York, USA) for analysis of non imaging data. Group comparisons were performed with ANOVA or Chi square. Comparison of mean FA and MD controlling for covariates was done with the general linear model, with fixed factors of group and sex, and covariates of age and deep WMH rating. Correlations performed with Pearson correlation.

\section{RESULTS}

Table 1 shows the subject demographics of the study participants with repeat DTI data. AD and DLB were well matched for overall cognitive impairment, though as expected, AD subjects performed worst on baseline episodic memory. The DLB subjects showed significant progression of motor features as assessed by increased UPDRS $(p=0.045)$. There were no significant differences between 
groups in WMH rating score. WMH score was significantly correlated $(p<0.001)$ with both mean FA and $\mathrm{MD}$ in the whole white matter at baseline, controlling for group and age.

Since a number of subjects had dropped out since our original baseline DTI study [3], we repeated analysis of the baseline FA and MD, but including only the subjects in the present report. The results from subjects with followup data were similar to those in the whole group, with FA decreased, and MD increased, in both AD and DLB (suppl. Figures e2 \& e3).

We found regions throughout the white matter where $M D$ increased longitudinally in AD relative to controls [figure 1]. There were also small regions in the left parietal and temporal lobe where the $M D$ increases in $A D$ were greater than DLB. No regions of significant change in MD were found in DLB or controls, or in FA in any group. Addition of covariates of age, sex and WMH rating did not qualitatively change these results. The mean increase in MD in the whole skeleton was significantly different between groups, (control 0.0044 SD $0.009 \times 10^{-3} \mathrm{~mm}^{2} \mathrm{~s}^{-1}$; AD $0.0139 \mathrm{SD} 0.016 \times 10^{-3} \mathrm{~mm}^{2} \mathrm{~s}^{-1}$ ; DLB 0.0056 SD $0.019 \times 10^{-3} \mathrm{~mm}^{2} \mathrm{~s}^{-1}$ ) $F_{2,66}=3.2, p=0.047$, with post hoc $A D>$ control (Tukey $p=0.045$ ). The group difference was still significant after including age, sex and $\mathrm{WMH}$ in the model $\left(\mathrm{F}_{2,63}=3.3\right.$, $p=0.038)$. Neither age, sex, nor baseline $\mathrm{WMH}$ rating significantly predicted change in MD ( $p>0.1)$. Overall change in mean $F A$ in the whole skeleton was not significantly different $\left(F_{2,66}=1.2\right.$, $\mathrm{p}=0.3$ ) between groups (Control -0.0005 SD 0.005; AD -0.0026 SD 0.0049; DLB -0.0009 SD 0.00589). Change in mean FA was still not significant between groups $(p=0.087)$ after the addition of age, sex and $\mathrm{WMH}$ rating to the model .

We investigated the correlation between change in whole skeleton FA \& MD and the percentage change in brain volume over the same period [9]. There was a significant correlation between brain atrophy and $M D$ change both in the whole group $(r=0.49 ; p<0.001)$ and the $A D(r=0.536 ; p=0.010)$ and control $(r=0.678 ; p<0.001)$ but not DLB $(r=0.07 ; p=0.8)$ groups. Change in FA did not significantly correlate with longitudinal brain atrophy in the whole group $(r=0.23 ; p=0.058)$, but was significant in the control $(r=0.39 ; p=0.026)$ but not $A D(r=0.05 ; p=0.8)$ or DLB $(r=-0.08 ; p=0.8)$. 
Within each group, we investigated the correlation between change in mean MD and FA, and change in cognitive measures (CAMCOG, MMSE, FAS, episodic memory) and UPDRS. Apart from decrease in CAMCOG, which correlated with decrease in FA in the $A D$ group $(r=0.47, p=0.023)$ there were no significant correlations.

\section{CONCLUSIONS}

We found increase of mean diffusivity over 1 year in the AD group to be greater than that in the control group over a wide range of brain areas. The increase in $M D$ in $A D$ was also greater than that in DLB in parietal and temporal regions. However, there was no evidence of longitudinal change in MD or FA in DLB relative to controls. This suggests that the widespread pattern of increased MD and reduced $F A$ in $A D$ that we saw at baseline is part of an ongoing degenerative process, whilst the alterations seen in DLB may have been an early marker of the disease, with less progression. However, we had only 1 year of followup, and relatively small alteration in cognitive score over the year, so it is possible that further change in MD and FA could occur as both diseases progress. Further study to investigate DTI from the earliest disease point would help clarify the disease courses.

We are aware of only a few studies investigating longitudinal changes in DTI in dementia. One study [13] investigated a mixed group of dementia (including $8 \mathrm{AD}$ ) and found regional changes in MD correlating with declining language function. That study used a VBM approach with smoothed MD images, which may have increased the influence of brain atrophy (via partial volume of CSF) on the $M D$ values. A separate study used a region of interest approach to look at changes in MD and FA in mild cognitive impairment $(\mathrm{MCl})$ and $A D$ compared to controls. They found longitudinal increases in 
$\mathrm{MD}$ in the Alzheimer's and $\mathrm{MCl}$ group in the cingulum. Only the control group showed change in FA. However, a study using tractography of the uncinate fasciculus (UNC), the inferior longitudinal fasciculus (ILF) and the inferior occipitofrontal fasciculus (IOFF) in AD found only longitudinally increased FA in the UNC of AD.[14]In the AD group, we found changes in MD, but not FA. This is in keeping with other previous studies [15-17] which have found greater sensitivity of MD to detect differences in AD. Possible reasons for this include the relatively large voxel size, since $M D$ is more uniform across the brain than FA, and hence less affected by registration errors. Also, whilst loss of white matter fibres will invariably increase MD, the effect on FA will depend on the underlying arrangement of the fibres. $[15,18]$ We saw a relationship between change in FA and atrophy in only the control group. This may be due to vascular disease being the primary pathology in control subjects, and vascular damage to the white matter leading directly to both altered brain volume, and also change in diffusion parameters. In the dementia groups, there will be greater influence of other pathologies (eg amyloid) on brain volume through grey matter damage, which may have less direct influence on white matter diffusion parameters. In this study we only investigated changes to the white matter major tracts. Future work should consider diffusion changes in the cortical and subcortical grey matter.

We found very limited evidence of association between cognitive decline and change in diffusion parameters. This is in keeping with our previous finding in this group that whole brain atrophy rate did not correlate with decline in cognitive score [9]. Possible reasons for this include the relatively short period ( 1 year) between assessments, as well as retest variability in cognitive scores.

Presence of CSF in voxels can have an influence on MD due to the high diffusivity and proton density of water, and it is possible that the correlation between MD and atrophy that we see is caused by increased CSF in the voxel. However, previous work [19] found that the TBSS method was relatively insensitive to partial volume effects, since the voxels in the white matter skeleton tend to be away from the edges of the brain. Cell shrinkage or loss in the WM will contribute to overall brain atrophy, 
and there is evidence that change in WM in $A D$ is at least partly independent of GM changes, and that it is an important part of the AD pathogenesis.[2] The rate of brain atrophy in DLB is lower than $A D$ [1] which also suggests that widespread brain degeneration is less marked in DLB, with changes to specific neurotransmitter systems (such as cholinergic or dopaminergic) being more profound. The relationship between change in brain volume and increased white matter diffusivity in AD would also be consistent with white matter changes in AD being largely secondary to grey matter or neuronal loss, so reflecting Wallerian degeneration. In contrast, the lack of such an association in DLB is consistent with synaptic dysfunction rather than cell loss as a key feature of the disorder, possibly caused by presynaptic accumulation of oligimeric alpha-synuclein.[20]

In conclusion we found that whereas at baseline MD was increased in both $A D$ and $D L B$, there was a continued increase in MD over time only in the AD group, with the DLB showing no change in MD. This may be a result of early changes in the DLB group, and studies of people at risk of, or in the very early stages of DLB may help clarify the timescale of the disease process.

\section{ACKNOWLEDGEMENTS}

The study was funded by the Sir Jules Thorn Charitable Trust (grant ref: 05/JTA), the NIHR Biomedical Research Unit in Dementia and the Biomedical Research Centre awarded to Cambridge University Hospitals NHS Foundation Trust and the University of Cambridge, and the NIHR Biomedical Research Unit in Dementia and the Biomedical Research Centre awarded to Newcastle upon Tyne Hospitals NHS Foundation Trust and the Newcastle University. Elijah Mak was in receipt of a Gates Cambridge, PhD studentship. 


\section{References}

[1] Mak E, Su L, Williams GB, O'Brien JT. Neuroimaging characteristics of dementia with Lewy bodies. Alzheimers Research \& Therapy. 2014;6:18 doi: 10.1186/alzrt248

[2] Amlien IK, Fjell AM. Diffusion tensor imaging of white matter degeneration in Alzheimer's disease and mild cognitive impairment. Neuroscience. 2014;276:206-15 doi:

10.1016/j.neuroscience.2014.02.017

[3] Watson R, Blamire AM, Colloby SJ, Wood JS, Barber R, He J, et al. Characterizing dementia with Lewy bodies by means of diffusion tensor imaging. Neurology. 2012;79:906-14 doi:

10.1212/WNL.0b013e318266fc51

[4] McKhann G, Drachman D, Folstein M, Katzman R, Price D, Stadlan EM. Clinical diagnosis of Alzheimer's disease: Report of the NINCDS-ADRDA Work Group under the auspices of Department of Health and Human Services Task Force on Alzheimer's disease. Neurology. 1984;34:939-44

[5] McKeith IG, Dickson DW, Lowe J, Emre M, O'Brien JT, Feldman H, et al. Diagnosis and management of dementia with Lewy bodies: Third report of the DLB consortium. Neurology. 2005;65:1863-72 doi: 10.1212/01.wnl.0000187889.17253.b1

[6] Roth M, Huppert FA, Mountjoy CQ, Tym E. CAMDEX-R: Revised Cambridge Examination For Mental Disorders Of The Elderly. Cambridge, UK: Cambridge University Press; 1999.

[7] Fahn S, Elton R, Members of the UPDRS development commitee. Unified Parkinson's disease rating scale. In: Fahn S, Marsden CD, Calne DB, Goldstein M, editors. Recent Developments In Parkinson's Disease. Florham Park; NJ: MacMillan Healthcare Information; 1987. p. 153-63, 293-304. [8] Smith SM, Nichols TE. Threshold-free cluster enhancement:Addressing problems of smoothing, threshold dependence and localisation in cluster inference. Neurolmage. 2009;44:83-98 doi: 10.1016/j.neuroimage.2008.03.061

[9] Mak E, Su L, Williams GB, Watson R, Firbank M, Blamire AM, et al. Longitudinal assessment of global and regional atrophy rates in Alzheimer's disease and dementia with Lewy bodies.

Neurolmage: Clinical. 2015;7:456-62 doi: 10.1016/j.nicl.2015.01.017

[10] Su L, Blamire AM, Watson R, He J, Aribisala B, O'Brien JT. Tissue microstructure changes in dementia with Lewy bodies revealed by quantitative MRI. J Neurol. 2015;262:165-72 doi: 10.1007/s00415-014-7541-2

[11] McRobbie DW, Moore EAG, M.J. , Prince MR. MRI From picture to proton. Cambridge, UK: Cambridge University Press; 2003.

[12] Fazekas F, Chawluk JB, Alavi A, Hurtig HI, Zimmerman RA. MR Signal abnormalities at $1.5 \mathrm{~T}$ in Alzheimer's dementia and normal aging. Am J Neuroradiol. 1987;8:421-6

[13] Frings L, Dressel K, Abel S, Mader I, Glauche V, Weiller C, et al. Longitudinal cerebral diffusion changes reflect progressive decline of language and cognition. Psychiat Res - Neuroim.

2013;214:395-401 doi: 10.1016/j.pscychresns.2013.08.003

[14] Kitamura S, Kiuchi K, Taoka T, Hashimoto K, Ueda S, Yasuno F, et al. Longitudinal white matter changes in Alzheimer's disease: a tractography-based analysis study. Brain Res. 2013;1515:12-8 doi: 10.1016/j.brainres.2013.03.052

[15] Acosta-Cabronero J, Williams GB, Pengas G, Nestor PJ. Absolute diffusivities define the landscape of white matter degeneration in Alzheimer's disease. Brain. 2010;133:529-39 doi: 10.1093/brain/awp257

[16] Nir TM, Jahanshad N, Villalon-Reina JE, Toga AW, Jack CR, Weiner MW, et al. Effectiveness of regional DTI measures in distinguishing Alzheimer's disease, $\mathrm{MCl}$, and normal aging. Neurolmage:

Clinical. 2013;3:180-95 doi: 10.1016/j.nicl.2013.07.006 
[17] Falangola MF, Jensen JH, Tabesh A, Hu C, Deardorff RL, Babb JS, et al. Non-Gaussian diffusion $\mathrm{MRI}$ assessment of brain microstructure in mild cognitive impairment and Alzheimer's disease. Magn Reson Imaging. 2013;31:840-6 doi: 10.1016/j.mri.2013.02.008

[18] Douaud G, Jbabdi S, Behrens TEJ, Menke RA, Gass A, Monsch AU, et al. DTI measures in crossing-fibre areas: Increased diffusion anisotropy reveals early white matter alteration in $\mathrm{MCl}$ and mild Alzheimer's disease. Neurolmage. 2011;55:880-90 doi: 10.1016/j.neuroimage.2010.12.008 [19] Berlot R, Metzler-Baddeley C, Jones DK, O'Sullivan MJ. CSF contamination contributes to apparent microstructural alterations in mild cognitive impairment. Neurolmage. 2014;92:27-35 doi: 10.1016/j.neuroimage.2014.01.031

[20] Schulz-Schaeffer WJ. The synaptic pathology of alpha-synuclein aggregation in dementia with Lewy bodies, Parkinson's disease and Parkinson's disease dementia. Acta Neuropathol.

2010;120:131-43 doi: 10.1007/s00401-010-0711-0 


\section{Table Captions}

Table 1 Demographic characteristics of the subjects.

\section{FIGURE CAPTIONS}

Figure 1 Group differences in MD.

Significant voxels $(p<0.05)$ in the white matter skeleton corrected for multiple comparisons where increase in $M D$ in $A D>$ control (red-yellow) and $A D$ > DLB (blue-pink). The white matter skeleton is shown in green. Images are presented in radiological convention (left of image is right of brain)

Supplementary figure e-1 Images with FLAIR type contrast calculated from quantitative T1 and T2 maps. Left show image rated as $\mathrm{WMH}=0$ and right $\mathrm{WMH}=3$.

Supplementary figure e-2 Comparison of FA at baseline in the subjects included in the longitudinal analysis.

Showing $\mathrm{p}<0.05$ (corrected for multiple comparisons with threshold free cluster enhancement) where FA in control $>A D$ and control $>$ DLB. There were no regions where FA was significantly decreased in controls relative to dementia

Supplementary figure e-3 Comparison of MD at baseline in the subjects included in the longitudinal analysis. 
Showing $p<0.05$ (corrected for multiple comparisons with threshold free cluster enhancement) where $M D$ in $A D>$ control and $D L B$ > controls. There were no regions where MD was significantly increased in controls relative to dementia . 
Table 1 Demographic characteristics of the subjects.

\begin{tabular}{|c|c|c|c|c|}
\hline & Control (N=32) & $A D(N=23)$ & $\operatorname{DLB}(N=14)$ & \\
\hline Age & $76.6(5.3)$ & $76.8(5.7)$ & $77.2(8.0)$ & $F_{2,66}=0.1, p=1.0$ \\
\hline $\operatorname{Sex}(F: M) \% F$ & $13: 19(41 \%)$ & 9 : $14(49 \%)$ & $1: 13(7 \%)$ & $x^{2}=5.4 ; p=0.07$ \\
\hline Interscan duration in days & $372(12)$ & $378(16)$ & $379(19)$ & $F_{2,66}=1.5, p=0.2$ \\
\hline Baseline CAMCOG & $97.9(3.4)$ & $69.5(11.6)$ & $69.9(17.3)$ & $T_{35}=-0.1, p=0.9$ \\
\hline CAMCOG change & $0.7(2.5)$ & $-5.4(9.5)^{*}$ & $-5.8(10.3)$ & $\mathrm{T}_{34}=0.1, \mathrm{p}=0.9$ \\
\hline Baseline MMSE & $29.2(0.9)$ & $20.4(4.0)$ & $21.2(6.0)$ & $T_{35}=-0.5, p=0.6$ \\
\hline MMSE change & $0.1(1.0)$ & $-1.5(2.9) *$ & $-2.5(2.8)^{* *}$ & $\mathrm{~T}_{34}=0.9, \mathrm{p}=0.3$ \\
\hline Baseline Episodic memory & $0.24(4.0)$ & $-17.6(2.8)$ & $-12.9(5.7)$ & $T_{34}=-3.3, p=0.002$ \\
\hline \multicolumn{5}{|l|}{ z-score } \\
\hline Change in Episodic & $1.42(3.31) *$ & $1.15(2.54) *$ & $-0.94(4.33)$ & $\mathrm{T}_{29}=1.7 ; \mathrm{p}=0.1$ \\
\hline \multicolumn{5}{|l|}{ memory z-score } \\
\hline Baseline FAS & $41.6(11.4)$ & $32.4(14.0)$ & $22.6(12.5)$ & $\mathrm{T}_{34}=2.1, \mathrm{p}=0.044$ \\
\hline FAS change & $-2.0(9.4)$ & $-7.1(9.0) * *$ & $-3.7(5.4) *$ & $\mathrm{~T}_{32}=-1.2, \mathrm{p}=0.3$ \\
\hline Baseline UPDRS & $1.8(1.6)$ & $4.6(3.6)$ & 27.2 (7.9) & $F_{2,66}=190, p<0.001$ \\
\hline UPDRS change & $0.2(1.4)$ & $0.9(2.4)$ & $4.8(8.1)^{*}$ & $F_{2,66}=6.9, p=0.002$ \\
\hline Deep WMH rating score & $1.03(1.12)$ & $1.52(0.99)$ & $1.21(0.97)$ & $F_{2,66}=1.5, p=0.24$ \\
\hline
\end{tabular}

${ }^{*} \mathrm{p}<0.05 ;{ }^{* *} \mathrm{p}<0.01$ : Within group longitudinal change (one sample $\mathrm{t}$ test)

FAS = letter fluency for words beginning F, A, S. UPDRS = united parkinson's disease rating scale part 3 (motor component). CAMCOG = cambridge cognitive examination. MMSE = mini mental state exam. Note for the change in episodic memory, data were available on 21 AD and 10 DLB. CAMCOG and MMSE change, memory baseline and FAS baseline in 13 DLB, change in FAS score from 11 DLB 


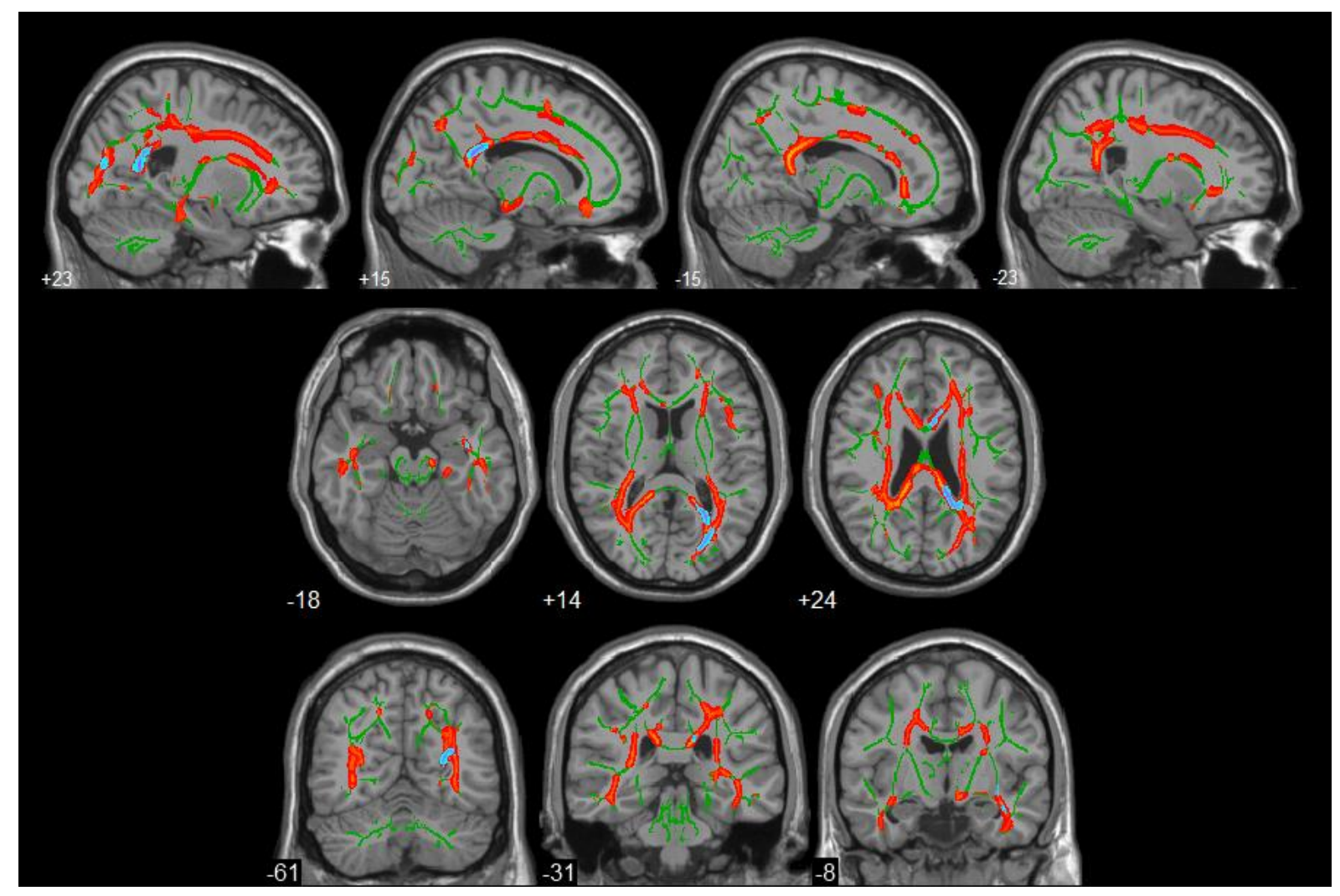


Longitudinal change in diffusion tensor imaging in dementia with Lewy bodies and Alzheimer's disease

Firbank et al

Supplementary figure 1 Images with FLAIR type contrast calculated from quantitative T1 and T2 maps. Left show image rated as $\mathrm{WMH}=0$ and right $\mathrm{WMH}=3$.

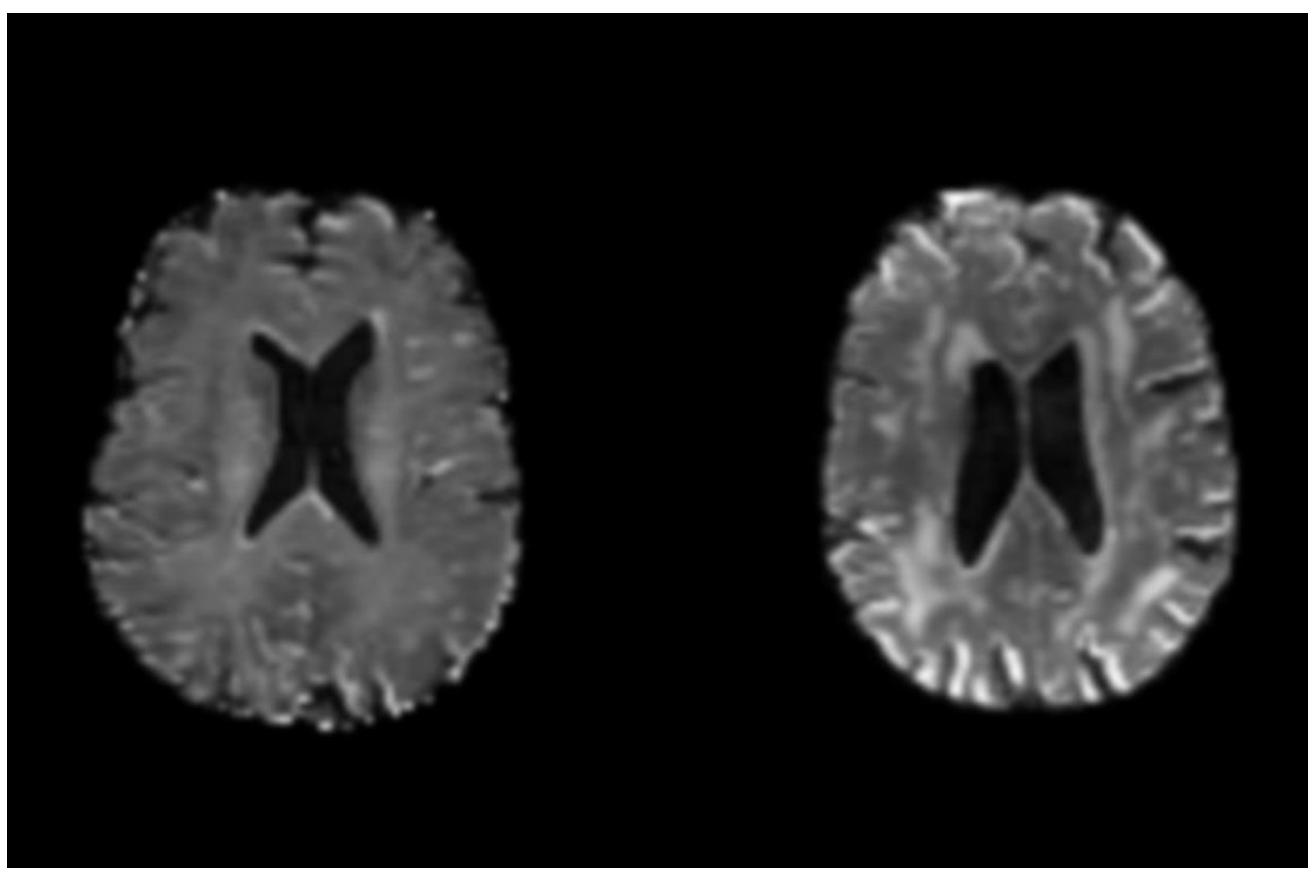


Supplementary figure 2 Comparison of FA at baseline in the subjects included in the longitudinal analysis. Showing $p<0.05$ (corrected for multiple comparisons with threshold free cluster enhancement) where FA in control > AD and control > DLB. There were no regions where FA was significantly decreased in controls relative to dementia

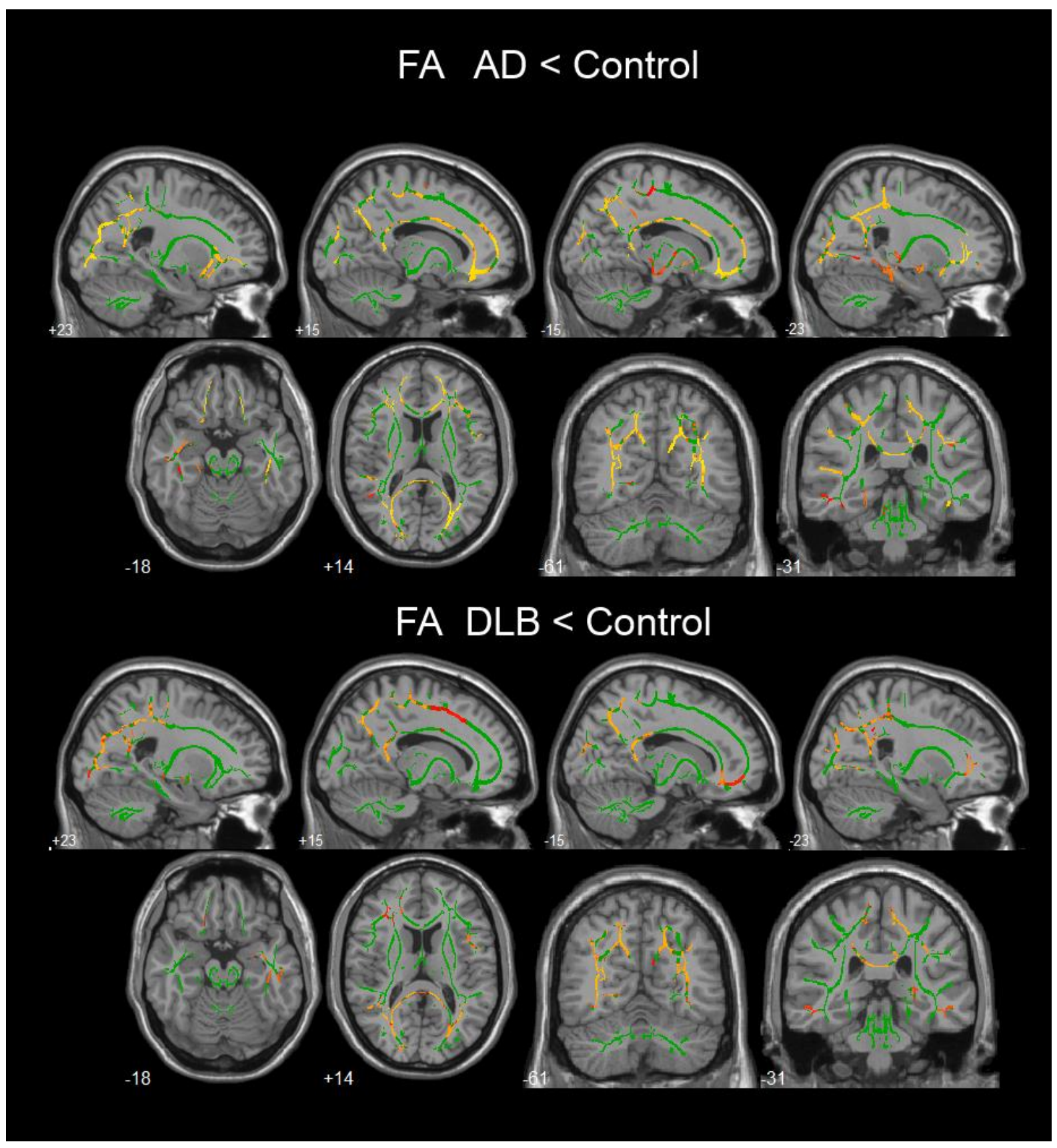


Supplementary figure 3 Comparison of MD at baseline in the subjects included in the longitudinal analysis. Showing $p<0.05$ (corrected for multiple comparisons with threshold free cluster enhancement) where $M D$ in $A D>$ control and $D L B$ > controls. There were no regions where MD was significantly increased in controls relative to dementia .

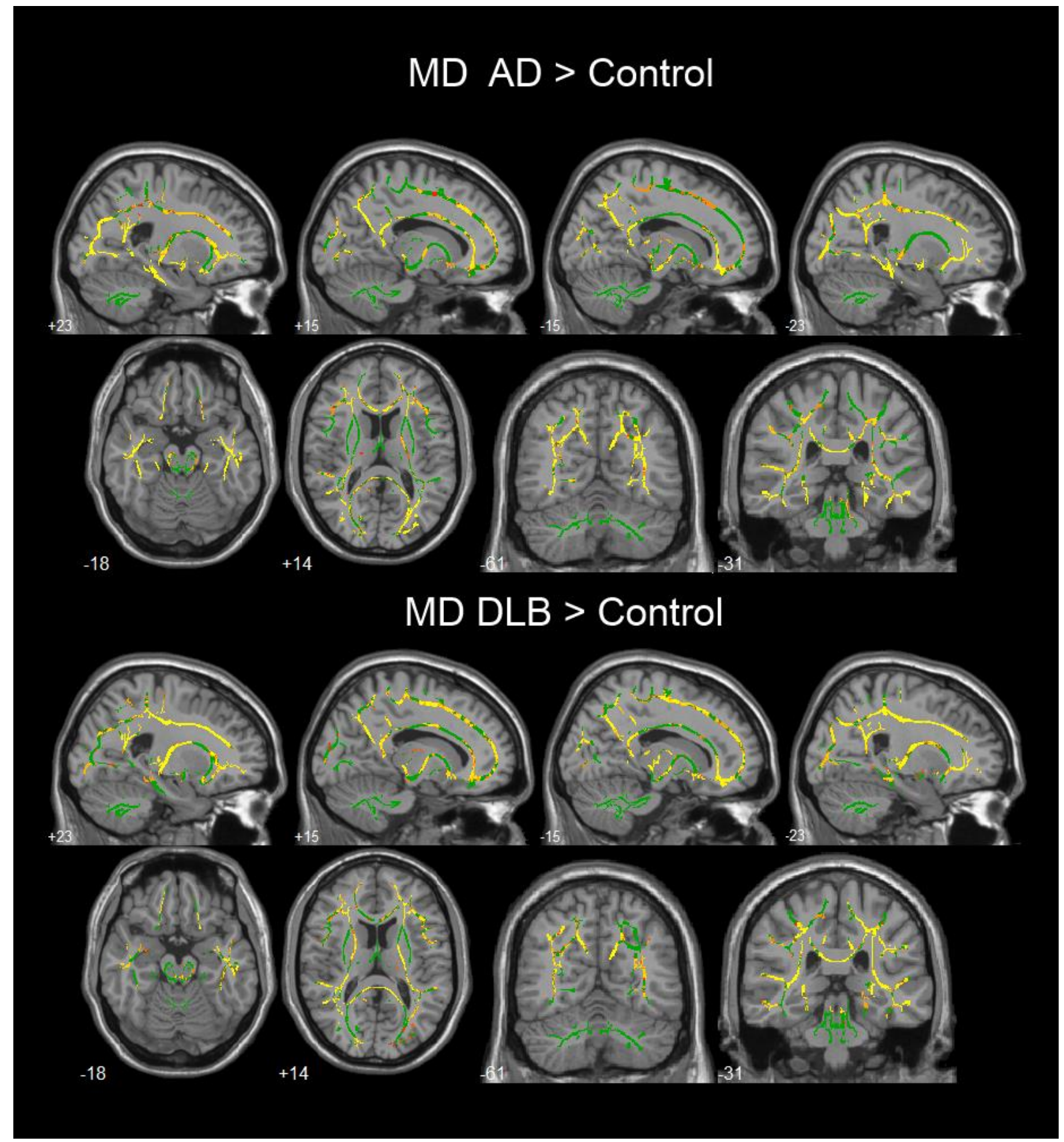

